\title{
ACQUISITION, PROCESSING, AND PRESENTATION OF 3-D CHROMATOVOLTAMMOGRAPHIC DATA USING AN IBM PS/2 AND PAR MODEL 273 POTENTIOSTAT
}

\author{
Samuel P. Kounaves* and David Daojng Lu \\ Department of Chemistry, Tufts University, Medford, MA 02155, U.S.A.
}

(Received 28 March 1991; in revised form 1 July 1991)

\begin{abstract}
Atstract-A software package for interfacing an IBM PS/2 and the EG\&G PAR model 273 potentiostat/galvanostat is described. The software is capable of controlling the instrument and taking and displaying both potential and current data simultaneously as a function of time (chromatovoltammetry). The software is written in both Microsoft QuickBASIC and QujekC for fast operation and easy portability. The software can also be easily modified to provide user-friendly control of the PAR 273. potentiostat for a multitude of other computer-controlled electrochemical experiments. An application is described for the determination of catecholamines using high-performance liquid chromatography with a carbon fiber ultramicroelectrode and square wave voltammetry for detection.
\end{abstract}

\section{INTRODUCTION}

The application of voltammetric fast-scan techniques to high-performance liquid chromatography (HPLC) has been investigated by several groups (Goto \& Shimada, 1986; White et al., 1986; Caudill et al., 1983). Recently we described the use of square wave voltammetry (SWV) at a carbon fiber ultramicroelectrode as an HPLC detector in the determination of biogenic amines (Kounaves \& Young, 1989). SWV is an electrochemical technique which allows the rapid scanning of a voltage range with a minimal contribution of charging current to the output signal (Osteryoung \& Osteryoung, 1985). Our system uses a commercially available potentiostat and microcomputer, and a custom fabricated working electrode consisting of a combination carbon fiber (permanently mounted at the HPLC column outlet on a glass slide) and flow-through cell. The system is used to obtain qualitative and quantitative data from a solution containing mixture of biogenic amines, with the results presented in the form of contour and isochromic plots (i.e. chromatovoltammograms). Combining SWV with HPLC provides a new "dimension" in the collection and analysis of chromatographic data in that both the chromatographic domain (time vs concentration signal) and the electrochemical domain (potential vs current) can be viewed simultaneously, allowing peaks and overlap in either technique to be resolved and detected separately.

This combination of techniques requires the rapid acquisition of electrochemical data through a computer controlled potentiostat and the real-time dis-

* Author for correspondence. play of the data in a 3-D form. Software packages currently supplied with commercially available electrochemical instrumentation offer the user extensive control with performing various voltammetric methods, however they do not offer the possibility of collecting both potential and current data simultaneously as a function of time. Our original software was written in Microsoft QuickBASIC and was recently also rewritten in Microsoft QuickC for faster operation and easier portability. In addition to the herein described use, the software can easily be modified to provide user-friendly control of the PAR 273 potentiostat for a multitude of other computercontrolled electrochemical experiments requiring 3-D or 2-D display of data.

\section{INSTRUMENTATION}

\section{Electrochemical and chromatographic}

The liquid chromatography system consisted of a model 196 mini-pump (Milton Roy), a $50 \mathrm{ml}$ loop injection valve (model 7125 , Rheodyne), a pulse dampener, and a $150 \times 4 \mathrm{~mm}$ Bio-Sil ODS.5S reversed-phase column (Rio-Rad). The mobile phase contained $0.1 \mathrm{M}$ sodium acetate (Fisher), $0.04 \mathrm{M}$ citric acid (Mallinckrodt), $0.8 \mathrm{mM}$ sodium octylsulfate (Kodak), $0.1 \mathrm{mM}$ EDTA (Fisher) and $5 \%$ methanol (Fisher), and was adjusted to a pH of 5.2 with sodium hydroxide.

The potentiometer used was the EG\&G Princeton Applied Research potentiostat/galvanostat model 273 (PAR 273). The PAR 273 can be operated as a stand-alone instrument using the front panel or under computer control through an RS232C serial or a GPIB-488 parallel data link. Control is accomplished through a command set of over 100 words, providing 
access to timing functions, real-time data averaging and current gain, in addition to all front panel functions. The working electrode consisted of a $7 \mu \mathrm{m}$ dia carbon fiber extended through a $0.8 \times 30 \mathrm{~mm}^{2}$ capillary tube (Kimax-51) and mounted with epoxy onto a glass slide on which Teflon capillary tubing had been mounted (Kounaves \& Young, 1989). The complete electrochemical detector consisted of the above electrode assembly mounted in a modified HDPE bottle along with a PT-wire counter electrode and saturated $\mathrm{NaCl}$ calomel reference electrode. All solutions were prepared with $18 \mathrm{M} \Omega$ watcr from an ion-exchange system (Millipore). Epinephrine (E), norepinephrine (NE), dopamine (DA), 5-hydroxytryptophan (5-HTP), 3,4-dihydroxybenzylamine (DHBA) and normetanephrine (NM), were obtained from Sigma. Standard solutions were made up containing $1.0 \mathrm{mM}$ of each amine of $0.1 \mathrm{M}$ perchloric acid and stored at $4^{\circ} \mathrm{C}$.

\section{Computer and interface hardware}

The PAR 273 was controlled by an IBM PS/2 model 30-286 with a $1024 \mathrm{~K}$ memory, a VGA color graphics adaptor, an 80287 math co-processor and a 30 Mbyte hard disk. Because of the need for real-time data acquisition and 3-D data display, the co-processor, a minimum of $1024 \mathrm{~K}$ memory and a 80286 of faster CPU is essential. A laser printer is useful for hard copies of the high resolution 3-D but a dotmatrix printer will also suffice for screen dumps of the isochromatic data.

The communication interface between the PAR 273 to the IBM PS/2 was provided by a National Instruments GPIB PC-2A (IEEE-488) interface card. This type of bit-parallel interface was used in order to provide the fastest possible data transfer and control.

\section{SOFTWARE}

The software has been written in both Microsoft QuickC and QuickBASIC. The source code in both cases is very similar and thus the description below applies equally well to both versions with exceptions noted. When compiled, the source code must be linked with the National Instruments GPIB interface object code routines. In general, the main program (3DHPLC) initializes the PAR 273, sets up all necessary parameters for the experiment, takes the data, displays it in real-time on the screen, allows limited manipulation of the $x / y$ data and can save to disk.

A hardcopy listing of the source code is available (at no cost) upon request from the authors, or both the source and executable code may be obtained by providing a low-density 5.25 or $3.5^{\prime \prime}$ floppy disk in a stamped self-addressed floppy mailer.

\section{Initialization and acquisition}

The initialization part of the main program begins by recognizing the attached devices using one of the
National Instruments GPIB function calls (National Instruments, 1989) to open the device and obtain the unit descriptor associated with the given name, sending the interface clear for $100 \mu \mathrm{s}$, checking the status and enabling direct memory access (DMA). It then sends various other commands to initialize the PAR 273 and set the proper parameters, loads the square wave into the PAR 273 memory, and turns on the cell. Next the computer timer is called, obtains current time and saves it for testing time expiration later, sends commands for "New Curve", "Take Curve", "Wait till Curve Done" and "Dump Data into the PAR 273 memory". At this point the current measurement data have been collected and stored in the PAR 273 memory.

Transferring the data from the PAR 273 to the PS/2 computer is accomplished by the ibrd (pstat, da, cnt) call. The "cnt" (count) bytes of data are transferred from the PAR 273 into the "da" data array. One key procedure at this point is to swap the pair of bytes representing a data point. This is necessary any time there is a data transfer from the PAR 273 to the host computer or from the host computer to the PAR 273 (e.g. loading the square wave to the PAR 273). The reason for swapping is that when the data are transferred in binary with 2 byte/point, the high-order byte of a given point is set first, followed by the lower-order byte, thus, they have to be switched back after the transfer. In this case of loading the square wave to the PAR 273, the data are swapped before the transfer.

To obtain the net differential current values for displaying (i.e. difference between the forward and reverse currents) we take the difference between the adjacent collected current data points, $\operatorname{cur}(j, i)=$ $\mathrm{da}(2 * i-1)-\mathrm{da}(2 * i)$, where $\mathrm{da}()$ is the data array read from the PAR 273 memory, " $j$ " is the scan number and " $i$ " is the measured current value. At the same time, the maximum and minumum values of the net differential current $\operatorname{array,} \operatorname{cur}(i, j)$, are also determined.

\section{Real-time screen display}

Immediately after the net differential current curve scan is calculated, each value for the scan is mapped into a color map using a total of 12 colors in the QuickC version or 15 colors in the QuickBASIC version. Each datum is mapped into one of the predefined color values since the maximum and minimum range of this particular scan is known. Since we already know the potential range (user defined before the experiment) and the total number of scans that will be made, the $X$ and $Y$ coordinates of the data viewport are uniquely predefined for each point. The data point is indicated on the screen by setting (turning on) the pixel at this specific position with the mapped color value. In the $C$ version, 3 pixels are set for each data point, itself and two adjacent pixels, one on the left, on the right. This gives a better resolution because the data viewport is a rectangle, with more 
space along the horizontal direction. Since the colors used during each scan are defined using the minimum and maximum for the scan, the maximum point for each scan will be the same color regardless of its relationship to other scans. This problem may be overcome in one of two ways, either by recalculating and redisplaying after each scan or by waiting till the end scan then recalculating and displaying. The first method provides a better display while the experiment is in progress, but will limit the scan rate at which the data can be collected. After evaluating both methods we chose the latter, i.e. after the last scan the entire current data array " $\operatorname{cur}(j, i)$ " is remapped according to the maximum and minimum found for the entire data set. If a decrease in the rate of data collection can be tolerated, the first method can be implemented by slightly reordering the algorithm.

\section{User-specified parameters}

The main menu allows the user to set several experiment parameters before each chromatographic run. The limitations imposed on the experimental parameters result from either the PAR 273 electronics or the software by way of computer memory and speed specifications.

Table 1 shows the input parameters and the range of values allowed. Some of these values are limited only by the computer speed and memory size, so they may be different depending on the type of computer used. For example, the run time and scan interval depend on the total number of scans stored, which in turn, depends on the amount of memory available for the data array. Other parameter limits such as the $S W$ frequency (scan rate), potential range or current range, are intrinsic functions of the PAR 273 potentiostat.

\section{Chromatographic sampling/scan interval}

Each potential range scan takes approx. 1 s. For example, a typical chromatographic experiment might take a total of $15 \mathrm{~min}$ resulting in about 150 scans. Assuming this time frame, the scan interval (the time between two scans) would be $(15 \times 60) \mathrm{s} / 150 \mathrm{scans}=6 \mathrm{~s} / \mathrm{scan}$. The program checks the time limit by taking the difference between current time (after one complete scan) and the time taken before this scan, compares it with the scan interval (user defined), waits until time expires, then takes the next curve.

\section{Data manipulation/analysis capability}

After finishing data acquisition, the results are displayed as an isochromic contour image on the screen. In the $\mathrm{C}$ version the operator can then directly determine at what potential range and time duration a peak occurs, and with the aid of a movable $x y$ crosshair can determine selected peak heights. In the QuickBASIC version the analysis section is a separate but similar program. A second viewport is provided to view transverse slices. In this viewport, the $X$ - or $Y$-slice of the image in the first viewport is displayed. The $X$-slice is horizontal slice of the image. It displays the current values vs time (scan numbers) at a particular potential valuc. The $Y$-slice is a vertical slice of the image. It displays the current values vs potential at a specific time (one scan).

\section{Data processing/numerical filtering}

As a result of the electronics, the electrode and the nA current levels measured, the collected data may contain a high level of noise. Thus, a data processing technique must be applied to filter the noise data. In the QuickBASIC version, a separate subprogram allows baseline subtraction and 1-D filtering to be done to either the $x$ or $y$ data. In the $C$ version a 2-D moving average filter (MAF) algorithm called Neighborhood Averaging (Gonzalez, 1987) is used to smooth the collected data contour image, and is implemented as a separate subprogram from the main. Given an $(n \times n)$ image as $f(x, y)$, the procedure is to generate a smoothed image $g(x, y)$ whose gray-level at every point is obtained by averaging the gray-level values of the pixels of $f(x, y)$ contained in predefined neighborhood $w(x, y)$ of the point $(x, y)$. In other words, the filter is a small square ( $m \times m$ pixels) moving window $w(x, y)$. It smooths the original image $f(x, y)$ first horizontally, then vertically. Eventually, it goes through the entire image and generates the smoother image $g(x, y)$. The user may determine the size of the moving window $w(x, y)$, i.e. the $m$ value, typically $m<10$. The user may also smooth an image again and again based on previous smoothing results until satisfactory resolution is obtained.

\section{Hardcopy output}

The resulting data from this program may be printed in two different ways. The most straightforward and fastest way is to do a graphics screen dump

Table 1. User-specifiable parameters and their ranges

\begin{tabular}{lll}
\hline \multicolumn{1}{c}{ Parameter } & \multicolumn{1}{c}{ Range } & \multicolumn{1}{c}{ Limiting factor } \\
\hline Maximum potential & $\pm 2500 \mathrm{mV}$ & PAR 273 electronics \\
Initial-final potential & $\leqslant 1000 \mathrm{mV}$ & Program array size \\
Step height & $>2 \mathrm{mV}$ & Program array size, electrochemical \\
Pulse amplitude & $10-100 \mathrm{mV}$ & Electrochemical \\
Square wave frequency & $10-5000$ & PAR 273 electronics \\
Current range & $4 \mathrm{nA}-1000 \mathrm{~mA}$ & PAR 273 electronics \\
No. of scaus & $>2$ & Program array size, scan interval \\
Scan interval & $\geqslant 2 \mathrm{~s}$ & Program array size, No. of scans \\
Post-inj. delay & $\geqslant 0 \mathrm{~s}$ & None \\
Precond. scans & $\geqslant 0$ & None \\
\hline
\end{tabular}


to a dot-matrix printer. For the IBM PS/2 this requires running under IBM DOS version 4.0 or later and loading the graphics module before pressing PRINT SCREEN. This procedure results in a grayscale printout of the isochromic display exactly as displayed on the screen. The resolution will depend on the type of dot-matrix printer used. For higher quality data presentation, the data saved with the program is formatted so that it can be imported to the commercially available SURFER ${ }^{\mathrm{TM}}$ software

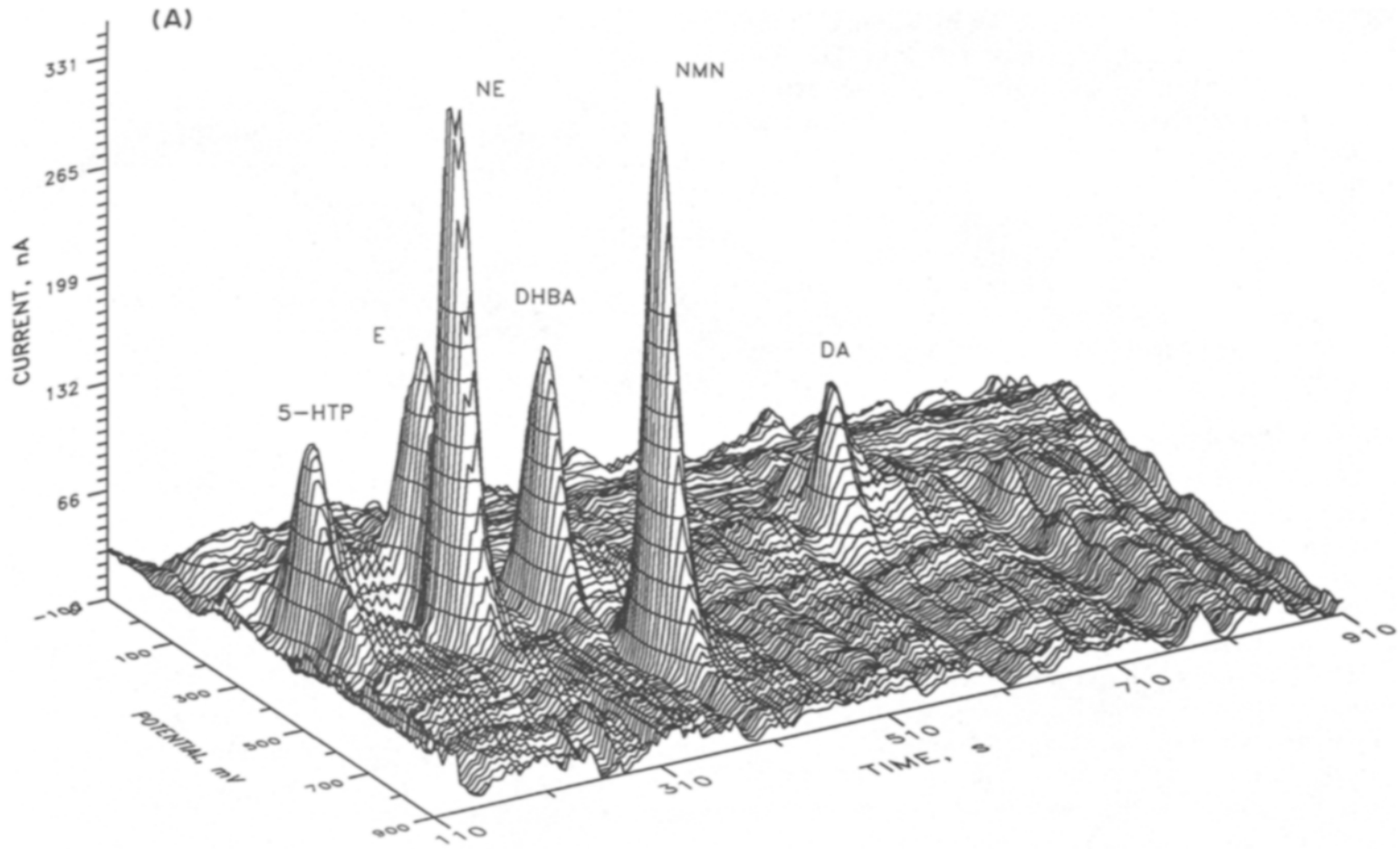

(B)

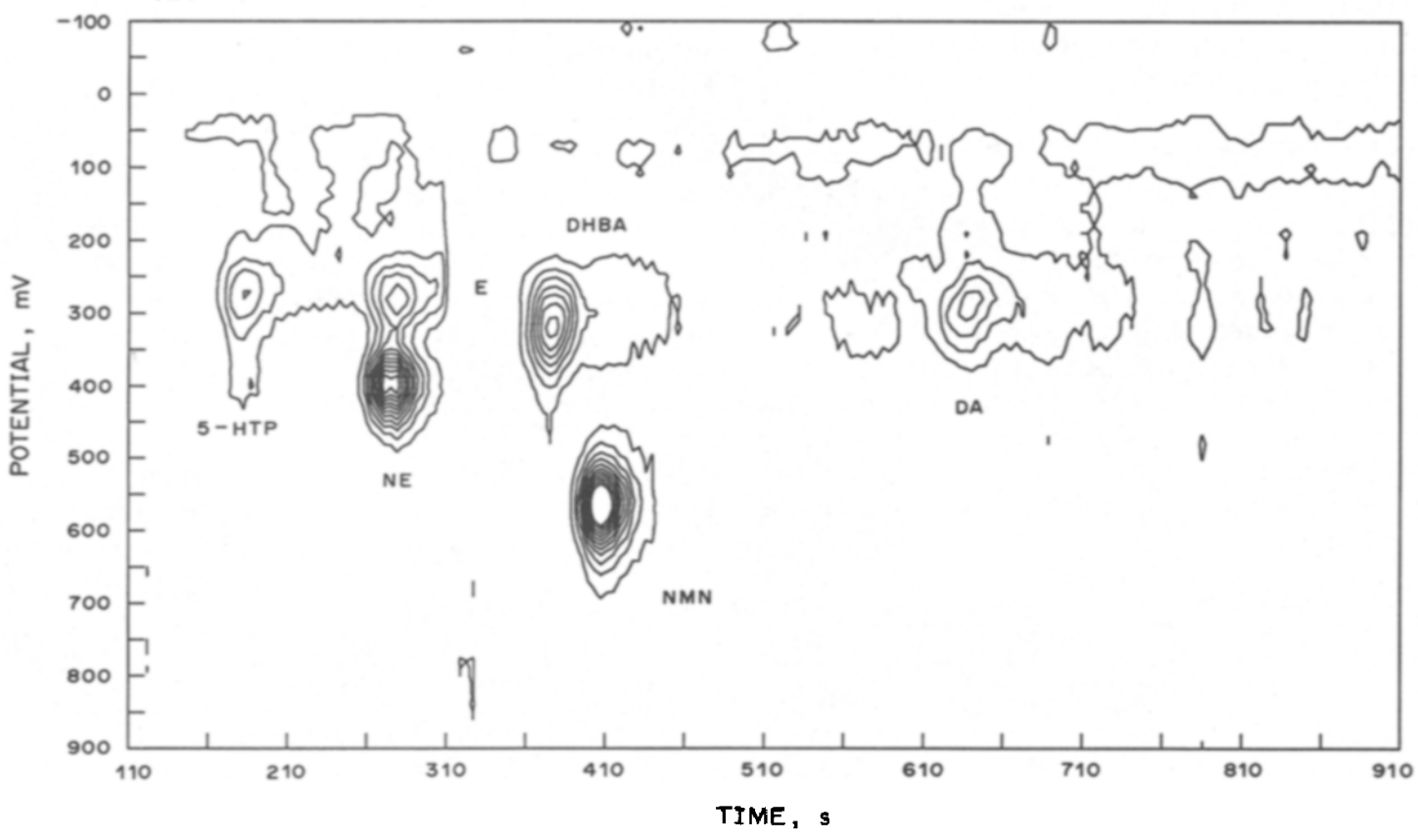

Fig. 1. Hardcopy output (using the SURFER software) of a square wave chromatovoltammogram of 5-HTP, E, NE, DHBA, NM and DA, all at $1 \times 10^{-6} \mathrm{M}$, represented as (A) a 3-D XYZ plot and as (B) an isogalvanic contour plot. Chromatographic and voltammetric conditions are given in the text. 


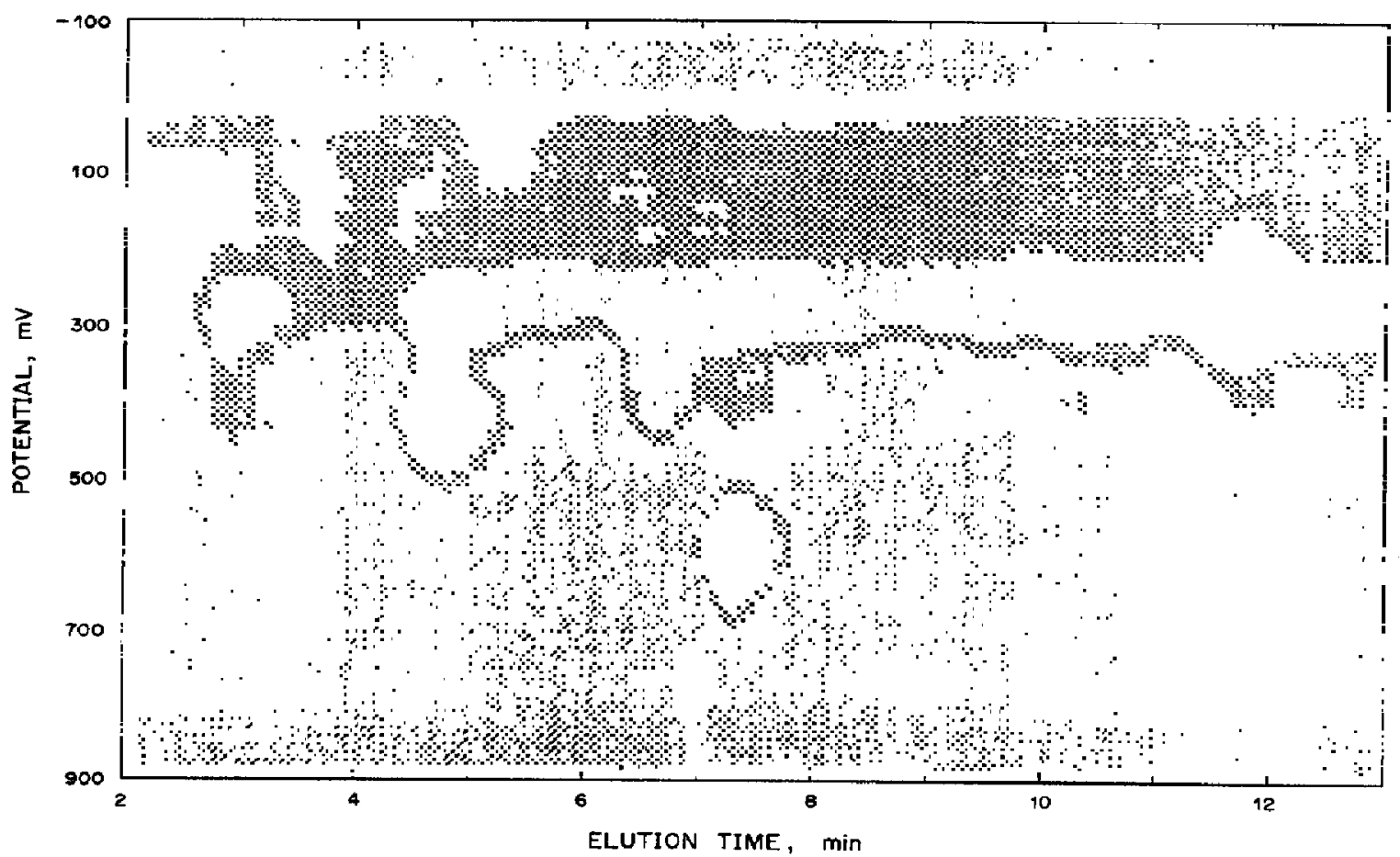

Fig. 2. A direct printer output of the 15-color isochromic contour plot as displayed on the screen.

package (Golden Software). Other 3-D plotting packages are also suitable, but the data must be customized to match gridding protocols of the particular software package.

\section{RESULTS}

The results shown in Figs 1 and 2 are for a $50 \mu 1$ injection containing 5-HTP, E, NE, DHBA, NM and $\mathrm{DA}$, all at $1 \times 10^{-6} \mathrm{M}$. The sample was injected onto the column with a mobile phase flow rate of $1 \mathrm{ml} / \mathrm{min}$. The SWV potential was scanned every $2 \mathrm{~s}$ from 0 to $800 \mathrm{mV}$ with a step height $\left(\Delta E_{\mathrm{s}}\right)$ of $5 \mathrm{mV}$, a square wave amplitude $\left(E_{\mathrm{s}}\right)$ of $25 \mathrm{mV}$ and a square wave frequency $(f)$ of $100 \mathrm{~Hz}$. The raw SWV dat collected consisted of forward $\left(i_{\mathrm{f}}\right)$ and reverse $\left(i_{\mathrm{r}}\right)$ current as a function of potential and elution time. The data shown is the resulting net current $\left(i_{\mathrm{n}}=j_{\mathrm{f}-i_{\mathrm{r}}}\right)$ after background subtraction and smoothing. The 3-D chromatovoltammograph in Fig. 1(A) and the contour plot in Fig. 1(B) were obtained by exporting the smoothed data to SURFER ${ }^{\mathrm{TM}}$ and plotting an HP laserjet printer. Figure 1(A) is really only useful for a "qualitative feeling" of the results, with all of the biogenic amines in the sample being clearly resolved and quantifiable when the data is plotted in the form of a contour plot, as in Fig. 1(B). The same data is shown as a dot-matrix printer screen dump in Fig. 2. On the actual screen the colors are selected so as to clearly resolve and indicate the top of the individual peaks.

\section{CONCLUSION}

We have described here a relatively short and simple software package for the PAR 273 potentiostat, capable of controlling the instrument and taking and displaying 3-D chromatovoltammographic data. The programs, being written in two widely available languages, can be easily modified to run other types of computer-controlled electrochemical experiments involving the acquisition of either all three parameters (time, current and potential) or any two.

\section{REFERENCES}

Gaudill W. L., Ewing A. G., Jones S. \& Wightman R. M. (1963) Anal. Chem. 55, 1877.

Gonzalez R. C. (1987) Digital Image Processing. AddisonWesley, Reading, MA.

Goto M. \& Shimada K. (1986) Chromatographia 21, 631.

Kounaves S. P. \& Young J. B. (1989) Anal. Chem. 61, 1469.

National Instruments (1989) The IEEE-488 \& C Language Interfaces. National Instruments, Austin, TX.

Osteryoung J. \& Osteryoung R. A. (1985) Anal. Chem. 57, $101 \mathrm{~A}$.

White J. G., St. Clair R. L. \& Jorgenson J. W. (1986) Anal. Chem. 58, 293. 